\title{
A comparison of the risk factors of intrahepatic recurrence, early recurrencen, and multiple recurrences after resection for single nodular hepatocellular carcinoma
}

\author{
Hyun Joon An, Woo Young Shin, Keon-Young Lee, and Seung-lk Ahn \\ Department of Surgery, Inha University School of Medicine, Incheon, Korea
}

\begin{abstract}
Backgrounds/Aims: Intrahepatic recurrence is one of the most important causes of compromised prognosis after surgical resection of hepatocellular carcinoma (HCC). This retrospective study was designed to identify and compare the risks of recurrence, early recurrence and multiple recurrences in a single patient population. Methods: A series of 92 consecutive patients, who received resection for single nodular HCC at our institute from January 2007 to December 2013, were enrolled in this study. The patients were divided into recurrent and non-recurrent groups; the recurrent group was further divided into subgroups by applying two criteria: early and late recurrence (with a cutoff of 18 months), and single and multiple $(\geq 2)$ recurrence. The potential risk factors were compared using univariate and multivariate analyses. The subgroup analysis was performed to determine the effects of different cut-off values on the analysis. Results: 41 recurrences $(44.6 \%)$ occurred during a mean follow-up of 42.4 months. The Child-Pugh score, and the portal vein invasion were found to be independent risk factors of recurrence, but differentiation was the only independent risk factor of early recurrence. The serum alpha-fetoprotein, tumor size, tumor necrosis, and hemorrhage were found to be the risk factors of multiple recurrences according to the univariate analysis, but lacked significance according to the multivariate analysis. When the cutoffs for early and multiple recurrences were changed to $\leq 10$ months and $>3$ nodules, respectively, different risk factors were identified. Conclusions: Our results implicated that different factors can predict the recurrence, timing, and multiplicity of an HCC recurrence. Further studies should be conducted to prove the complex relationships between tumor burden, invasiveness, and underlying liver cirrhosis for initial tumors, and the timing and multiplicity of recurrent HCC. (Korean J Hepatobiliary Pancreat Surg 2015;19:89-97)
\end{abstract}

Key Words: Risk factors; Recurrence pattern; Hepatocellular carcinoma; Resection

\section{INTRODUCTION}

Surgical resection is the best management option, in patients with hepatocellular carcinoma (HCC), provided that the remnant liver function is adequate. ${ }^{1,2}$ However, intrahepatic recurrence is a major cause of prognosis compromise in HCC, even after liver transplantation. ${ }^{3,4}$ Hence, extensive studies have been performed to identify the risk factors that predict HCC recurrence. The studies conducted to date have stratified the patients into recurrent and non-recurrent groups, ${ }^{4-6}$ or according to the location of the recurrence (intrahepatic vs. extrahepatic), ${ }^{3,4}$ time to recurrence (early vs. late), ${ }^{5,7-9}$ or number of detected recurrent nodules (single vs. multiple vs. diffuse). ${ }^{10-12}$ The identification of factors related to recurrence is important for predicting the prognosis and for establishing treatment plans. ${ }^{10}$ However, published factor analysis results are often inconsistent, presumably due to different patient populations and small cohort sizes.

The aim of this study was to identify the risk factors of the intrahepatic recurrence of single nodular HCC, for all recurrences, whether early or multiple, and to compare the results in order to identify the common denominators of poor prognoses. To avoid pathologic uncertainty, we included only patients that underwent surgical resection as an initial management. In addition, the patients were restricted to single nodular HCC at presentation and intrahepatic recurrence only, to avoid confounding variables. ${ }^{13}$

Received: August 1, 2015; Revised: August 10, 2015; Accepted: August 15, 2015

Corresponding author: Keon-Young Lee

Department of Surgery, Inha University School of Medicine, 7-206, 3-ga Sinheung-dong, Jung-gu, Incheon 22332, Korea Tel: +82-32-890-3432, Fax: +82-32-890-3549, E-mail: 196087@inha.ac.kr

Copyright (C) 2015 by The Korean Association of Hepato-Biliary-Pancreatic Surgery

This is an Open Access article distributed under the terms of the Creative Commons Attribution Non-Commercial License (http://creativecommons.org/ licenses/by-nc/4.0) which permits unrestricted non-commercial use, distribution, and reproduction in any medium, provided the original work is properly cited. Korean Journal of Hepato-Biliary-Pancreatic Surgery • pISSN: 1738-6349 - eISSN: 2288-9213 


\section{MATERIALS AND METHODS}

From January 2007 to December 2013, 125 consecutive patients were managed by surgical resection for pathologically proven single nodular $\mathrm{HCC}$ at our institution, and all were initially considered for this study, with the follow-up ending on December $31^{\text {st }}$, 2014. Of these 125 patients, 33 were excluded in accordance with our exclusion criteria (Table 1). Six (4.8\%) of the 33 patients had an extrahepatic recurrence, and one patient $(0.8 \%)$ was a perioperative mortality. Another eight (6.4\%) patients were excluded due to a concurrence of combined intrahepatic cholangiocarcinoma.

The medical records of the remaining 92 were reviewed retrospectively, and constituted the study cohort. The operative methods were classified as hemihepatectomy, sectionectomy, segmentectomy (anatomical resection), and tumorectomy (non-anatomical resection). Regarding the pathologic data, the gross type was classified as described by The Korean Liver Cancer Study Group, and the differentiation was determined by the worst Edmondson-Steiner grade. ${ }^{9}$ Follow-ups were performed at 3 to 6 months intervals, unless a postoperative complication was encountered, or a recurrence was suspected. Follow-up studies and tests consisted of an alpha-fetoprotein (AFP) and a PIVKA (protein induced by vitamin $\mathrm{K}$ absence or antagonist), an abdominal computed tomography (CT) and/or magnetic resonance (MR) imaging, as well as a liver function test with coagulation profiles.

Recurrence was defined as the first appearance of an enhancing liver mass, with delayed wash-out, during the follow-up as determined by imaging studies. The patients were divided into recurrent and non-recurrent groups; the recurrent group was further divided into subgroups by ap- plying two criteria: namely, early or late recurrence, and single or multiple recurrences. Early recurrence was defined as the detection of the first recurrence within 18 months of surgery, which was approximated as the mean recurrence-free period (RFP) of all recurred patients. A risk factor analysis was performed to investigate the effect of time-to-recurrence by re-performing the analysis for a RFP of 10 months (the median RFP of all recurred patients). Multiple recurrences were defined as the detection of two or more recurrent nodules, or diffuse recurrence at first detection. Similarly, the analysis was re-performed by dividing the recurrent group by the nodule number, using cutoffs of 1 (single vs. multiple) or 3 (fewer, $\leq 3$ vs. diffuse, $>3$ ) to determine whether the choice of cutoff affects the results of the risk factor analysis. A clinicopathological factor analysis was performed using the Student's T-Test, the Chi-Square Test, or Fisher's Exact Test, as appropriate, to compare the groups. A multivariate analysis was performed using the Cox proportional hazard model for recurrence, to determine the statistical significances of factors identified by the univariate analysis, and a logistic regression analysis was used to identify factors associated with early and multiple recurrences. Median RFPs were calculated using the Kaplan-Meier method, and a factor analysis was performed using the log-rank test. Statistical analyses were performed using IBM SPSS Statistics for Windows, Version 19.0 (IBM Corp. Armonk, NY, USA), and the $p$-values of $<0.05$ were considered statistically significant.

\section{RESULTS}

\section{Patient characteristics}

The demographic data and clinicopathological parame-

Table 1. Inclusion and exclusion criteria

Inclusion criteria $(\mathrm{n}=92,73.6 \%)$

Pathologically proven HCC

Single nodular tumor without satellite nodule

Surgical resection as initial management
Exclusion criteria $(\mathrm{n}=33,26.4 \%)$

TACE or RFA as initial management $(n=8,6.4 \%)$

Coincident with other malignancies $(\mathrm{n}=8,6.4 \%)$

Extrahepatic recurrence $(n=6,4.8 \%)$

Follow-up loss immediately after operation $(n=5,4.0 \%)$

Liver transplantation $(\mathrm{n}=3,2.4 \%)$

Ruptured HCC ( $\mathrm{n}=2,1.6 \%)$

Perioperative mortality $(\mathrm{n}=1,0.8 \%)$

HCC, hepatocellular carcinoma; TACE, transarterial chemoembolization; RFA, radiofrequency ablation 
ters of the 92 patients are presented in Table 2. The overall mean patient age was 55.2 years, and there were 78 men and 14 women (a male to female sex ratio of 5.6:1). The most common cause of background liver diseases were hepatitis $B$ infection $(n=67)$. In contrast, 15 patients (16.3\%) had non-cirrhotic livers. In 76 patients $(82.7 \%)$, one or more liver segments were resected, and in 16 patients (17.4\%), only the HCC mass was removed by way of partial hepatectomy, with as much safety margin as possible.

\section{Risk factor analysis for tumor recurrence}

Over a mean follow-up of 42.4 months, 41 patients (44.6\%) were found to have an intrahepatic recurrence of HCC (Table 2). The mean follow-up periods in the non-re- currence and recurrence groups were not different (41.5 vs. 43.6 months, respectively, $p=0.695$ ). Of the parameters tested, the Child-Pugh score $(p=0.019)$, the maximum tumor diameter $(p=0.003)$, tumor necrosis $(p=0.022)$, portal vein invasion $(p=0.006)$, and pathologic tumor $(\mathrm{pT})$ stage [according to the $7^{\text {th }}$ AJCC staging system $(p=0.001)$ ] were found to be significantly associated with an HCC recurrence according to the univariate analysis. The multivariate analysis showed that the Child-Pugh score ( $p=0.001)$, portal vein invasion $(p=0.002)$, and the $\mathrm{pT}$ stage $(p=0.001)$ independently predicted recurrence (Table 3$)$. The RFP curve, according to pT stages, is shown in Fig. 1.

Risk factor analysis for early tumor recurrence Of the 41 recurred patients, 29 (70.7\%) recurred within

Table 2. Comparisons of the demographic data and clinicopathological factors of single nodular hepatocellular carcinoma patients, with or without recurrence

\begin{tabular}{|c|c|c|c|c|c|c|c|}
\hline \multirow[b]{2}{*}{ Factors } & \multirow[b]{2}{*}{ Overall $(\mathrm{n}=92)$} & \multirow[b]{2}{*}{$\begin{array}{c}\text { Without } \\
\text { recurrence } \\
(n=51,55.4 \%)\end{array}$} & \multicolumn{5}{|c|}{ With recurrence $(n=41,44.6 \%)$} \\
\hline & & & Overall & $\begin{array}{c}\text { Early } \\
(\leq 18 \mathrm{mos}) \\
(\mathrm{n}=29,70.7 \%)\end{array}$ & $\begin{array}{c}\text { Late } \\
(>18 \text { mos }) \\
(n=12,29.3 \%)\end{array}$ & $\begin{array}{c}\text { Single } \\
(n=31,75.6 \%)\end{array}$ & $\begin{array}{c}\text { Multiple } \\
(\mathrm{n}=10,24.4 \%)\end{array}$ \\
\hline Age (mean $\pm \mathrm{SD}$, years) & & & 0.494 & & 0.760 & & 0.198 \\
\hline & $55.2 \pm 9.6$ & $55.8 \pm 10.4$ & $54.4 \pm 8.5$ & $54.2 \pm 8.6$ & $55.1 \pm 8.6$ & $55.4 \pm 8.2$ & $51.4 \pm 9.2$ \\
\hline $\operatorname{Sex}(n)$ & & & 0.469 & & 0.620 & & 0.310 \\
\hline Male & $78(84.8 \%)$ & $42(82.4 \%)$ & $36(87.8 \%)$ & $26(89.7 \%)$ & $10(83.3 \%)$ & $26(83.9 \%)$ & $10(100 \%)$ \\
\hline Female & $14(15.2 \%)$ & $9(17.6 \%)$ & $5(12.2 \%)$ & $3(10.3 \%)$ & $2(16.7 \%)$ & $5(16.1 \%)$ & $0(0 \%)$ \\
\hline Follow-up period & & & 0.695 & & 0.000 & & 0.466 \\
\hline (Mean $\pm \mathrm{SD}, \mathrm{mos})$ & $42.4 \pm 25.0$ & $41.5 \pm 21.5$ & $43.6 \pm 29.0$ & $32.7 \pm 23.6$ & $70.1 \pm 23.9$ & $45.5 \pm 28.7$ & $37.7 \pm 30.9$ \\
\hline Background liver disease (n) & & & 0.613 & & 0.599 & & 0.385 \\
\hline HBV & $67(72.8 \%)$ & $37(72.5 \%)$ & $30(73.2 \%)$ & $21(72.4 \%)$ & $9(75.0 \%)$ & $24(77.4 \%)$ & $6(60.0 \%)$ \\
\hline $\mathrm{HCV}$ & $3(3.3 \%)$ & $1(2.0 \%)$ & $2(4.9 \%)$ & $2(6.9 \%)$ & 0 & $2(6.5 \%)$ & 0 \\
\hline ALD & $7(7.6 \%)$ & $3(5.9 \%)$ & $4(9.8 \%)$ & $2(6.9 \%)$ & $2(16.7 \%)$ & $2(6.5 \%)$ & $2(20.0 \%)$ \\
\hline Non-cirrhotic & $15(16.3 \%)$ & $10(19.6 \%)$ & $5(12.2 \%)$ & $4(13.8 \%)$ & $1(8.3 \%)$ & $3(9.7 \%)$ & $2(20.0 \%)$ \\
\hline MELD score & & & 0.759 & & 0.448 & & 0.575 \\
\hline$(\mathrm{Mean} \pm \mathrm{SD})$ & $7.9 \pm 1.5$ & $7.9 \pm 1.3$ & $7.8 \pm 1.6$ & $8.0 \pm 1.3$ & $7.7 \pm 1.2$ & $7.8 \pm 1.2$ & $8.1 \pm 1.4$ \\
\hline Child-Pugh Score (n) & & & 0.019 & & 0.242 & & 0.376 \\
\hline 5 & $78(84.8 \%)$ & $48(94.1 \%)$ & $30(73.2 \%)$ & $21(72.4 \%)$ & $9(75.0 \%)$ & $21(67.7 \%)$ & $9(90.0 \%)$ \\
\hline 6 & $13(14.1 \%)$ & $3(5.9 \%)$ & $10(24.4 \%)$ & $8(27.6 \%)$ & $2(16.7 \%)$ & $9(29.0 \%)$ & $1(10.0 \%)$ \\
\hline 7 & $1(1.1 \%)$ & 0 & $1(2.4 \%)$ & 0 & $1(8.3 \%)$ & $1(3.2 \%)$ & 0 \\
\hline $\mathrm{AFP}^{*}$ & & & 0.443 & & 0.037 & & 0.002 \\
\hline$(\mathrm{Mean} \pm \mathrm{SD}, \mathrm{ng} / \mathrm{ml})$ & $367.3 \pm 796.9$ & $424.8 \pm 959.3$ & $295.8 \pm 534.0$ & $375.4 \pm 609.3$ & $103.6 \pm 187.1$ & $386.0 \pm 587.8$ & $16.4 \pm 31.3$ \\
\hline PIVKA $* *$ & & & 0.248 & & 0.506 & & 0.350 \\
\hline$(\mathrm{Mean} \pm \mathrm{SD}, \mathrm{mAU} / \mathrm{ml})$ & $757.5 \pm 5349.8$ & $81.2 \pm 73.3$ & $1610.8 \pm 8025.1$ & $2173.3 \pm 9513.0$ & $230.0 \pm 214.6$ & $322.2 \pm 737.6$ & $5762.9 \pm 16448.1$ \\
\hline Operation (n) & & & 0.070 & & 0.102 & & 0.848 \\
\hline Hemihepatectomy & $11(12.0 \%)$ & $5(9.8 \%)$ & $6(14.6 \%)$ & $6(20.7 \%)$ & $0(0.0 \%)$ & $5(16.1 \%)$ & $1(10.0 \%)$ \\
\hline $\begin{array}{l}\text { Segmentectomy or } \\
\text { sectionectomy }\end{array}$ & $65(70.7 \%)$ & $33(64.7 \%)$ & $32(78.0 \%)$ & $22(75.9 \%)$ & $10(83.3 \%)$ & $24(77.4 \%)$ & $8(80.0 \%)$ \\
\hline Partial hepatectomy & $16(17.4 \%)$ & $13(25.5 \%)$ & $3(7.3 \%)$ & $1(3.4 \%)$ & $2(16.7 \%)$ & $2(6.5 \%)$ & $1(10.0 \%)$ \\
\hline
\end{tabular}

*Maximum value was set to $40,000 \mathrm{ng} / \mathrm{ml}$. **Maximum value was set to $2,000 \mathrm{mAU} / \mathrm{ml}$. ***Tumor staging according to $7^{\text {th }}$ AJCC tumor staging system. HBV, hepatitis B virus; HCV, hepatitis C virus; ALC, alcoholic liver disease; MELD, model for end-stage liver disease; AFP, alpha-fetoprotein; PIVKA, protein induced by vitamin $\mathrm{K}$ absence or antagonist 
Table 2. Continued

\begin{tabular}{|c|c|c|c|c|c|c|c|}
\hline \multirow[b]{2}{*}{ Factors } & \multirow[b]{2}{*}{ Overall $(n=92)$} & \multirow{2}{*}{$\begin{array}{c}\text { Without } \\
\text { recurrence } \\
(n=51,55.4 \%)\end{array}$} & \multicolumn{5}{|c|}{ With recurrence $(n=41,44.6 \%)$} \\
\hline & & & Overall & $\begin{array}{c}\text { Early } \\
(\leq 18 \text { mos }) \\
(n=29,70.7 \%)\end{array}$ & $\begin{array}{c}\text { Late } \\
(>18 \text { mos }) \\
(n=12,29.3 \%)\end{array}$ & $\begin{array}{c}\text { Single } \\
(n=31,75.6 \%)\end{array}$ & $\begin{array}{c}\text { Multiple } \\
(\mathrm{n}=10,24.4 \%)\end{array}$ \\
\hline Resection margin & & & 0.412 & & 0.799 & & 0.721 \\
\hline$(\mathrm{Mean} \pm \mathrm{SD}, \mathrm{cm})$ & $0.55 \pm 0.60$ & $0.60 \pm 0.66$ & $0.50 \pm 0.54$ & $0.51 \pm 0.61$ & $0.46 \pm 0.32$ & $0.48 \pm 0.53$ & $0.55 \pm 0.59$ \\
\hline Margin involvement (n) & & & 0.459 & & 1.000 & & 1.000 \\
\hline No & $84(91.3 \%)$ & $48(94.1 \%)$ & $36(87.8 \%)$ & $25(86.2 \%)$ & $11(91.7 \%)$ & $27(87.1 \%)$ & $9(90.0 \%)$ \\
\hline Yes & $8(8.7 \%)$ & $3(5.9 \%)$ & $5(12.2 \%)$ & $4(13.8 \%)$ & $1(8.3 \%)$ & $4(12.9 \%)$ & $1(10.0 \%)$ \\
\hline \multicolumn{8}{|l|}{ Pathologic parameters } \\
\hline $\begin{array}{l}\text { Maximum diameter } \\
\quad(\text { Mean } \pm \mathrm{SD}, \mathrm{cm})\end{array}$ & $3.5 \pm 2.5$ & $2.8 \pm 1.2$ & $\begin{array}{l}0.003 \\
4.5 \pm 3.3\end{array}$ & $4.9 \pm 3.7$ & $\begin{array}{l}0.268 \\
3.6 \pm 2.0\end{array}$ & $3.8 \pm 2.6$ & $\begin{array}{l}0.017 \\
6.6 \pm 4.5\end{array}$ \\
\hline Gross type (n) & & & 0.075 & & 0.793 & & 0.303 \\
\hline Expanding nodular & $45(48.9 \%)$ & $20(39.2 \%)$ & $25(61.0 \%)$ & $17(58.6 \%)$ & $8(66.7 \%)$ & $20(64.5 \%)$ & $5(50.0 \%)$ \\
\hline Multinodular confluent & $23(25.0 \%)$ & $14(27.5 \%)$ & $9(22.0 \%)$ & $6(20.7 \%)$ & $3(25.0 \%)$ & $7(22.6 \%)$ & $2(20.0 \%)$ \\
\hline Nodular & $23(25.0 \%)$ & $17(33.3 \%)$ & $6(14.6 \%)$ & $5(17.2 \%)$ & $1(8.3 \%)$ & $4(12.9 \%)$ & $2(20.0 \%)$ \\
\hline Infiltrative & $1(1.1 \%)$ & 0 & $1(2.4 \%)$ & $1(3.4 \%)$ & $0(0.0 \%)$ & $0(0.0 \%)$ & $1(10.0 \%)$ \\
\hline Worst differentiation, No. & & & 0.289 & & 0.003 & & 0.892 \\
\hline Edmondson grade I & 0 & 0 & 0 & 0 & 0 & 0 & 0 \\
\hline Edmondson grade II & $14(15.2 \%)$ & $10(19.6 \%)$ & $4(9.8 \%)$ & $1(3.4 \%)$ & $3(25.0 \%)$ & $3(9.7 \%)$ & $1(10.0 \%)$ \\
\hline Edmondson grade III & $42(45.7 \%)$ & $24(47.1 \%)$ & $18(43.9 \%)$ & $10(34.5 \%)$ & $8(66$. & $13\left(41.9^{\circ}\right.$ & $5(50.0 \%)$ \\
\hline Edmondson grade IV & $36(39.1 \%)$ & $17(33.3 \%)$ & $19(46.3 \%)$ & $18(62.1 \%)$ & $1(8.3 \%)$ & $15(48.4 \%)$ & $4(40.0 \%)$ \\
\hline Tumor necrosis (n) & & & 0.022 & & 0.380 & & 0.012 \\
\hline No & $63(68.5 \%)$ & $40(78.4 \%)$ & $23(56.1 \%)$ & $15(51.7 \%)$ & $8(66.7 \%)$ & $21(67.7 \%)$ & $2(20.0 \%)$ \\
\hline Yes & $29(31.5 \%)$ & $11(21.6 \%)$ & $18(43.9 \%)$ & $14(48.3 \%)$ & $4(33.3 \%)$ & $10(32.3 \%)$ & $8(80.0 \%)$ \\
\hline Hemorrhage (n) & & & 0.069 & & 0.730 & & 0.022 \\
\hline No & $67(72.8 \%)$ & $41(80.4 \%)$ & $26(63.4 \%)$ & $19(65.5 \%)$ & $7(58.3 \%)$ & $23(74.2 \%)$ & $3(30.0 \%)$ \\
\hline Yes & $25(27.2 \%)$ & $10(19.6 \%)$ & $16(36.6)$ & $10(34.5 \%)$ & $5(41.7 \%)$ & $8(25.8 \%)$ & $7(70.0 \%)$ \\
\hline Capsule formation (n) & & & 0.655 & & 0.904 & & 0.994 \\
\hline No & $25(27.2 \%)$ & $13(25.5 \%)$ & $12(29.3 \%)$ & $9(31.0 \%)$ & $3(25.0 \%)$ & $9(29.0 \%)$ & $3(30.0 \%)$ \\
\hline Partial & $43(46.7 \%)$ & $26(51.0 \%)$ & $17(41.5 \%)$ & $12(41.4 \%)$ & $5(41.7 \%)$ & $13(41.9 \%)$ & $4(40.0 \%)$ \\
\hline Complete & $24(26.1 \%)$ & $12(23.5 \%)$ & $12(29.3 \%)$ & $8(27.6 \%)$ & $4(33.3 \%)$ & $9(29.0 \%)$ & $3(30.0 \%)$ \\
\hline Capsule infiltration (n) & & & 0.869 & & 0.431 & & 0.484 \\
\hline No & $48(52.2 \%)$ & $27(52.9 \%)$ & $21(51.2 \%)$ & $16(55.2 \%)$ & $5(41.7 \%)$ & $17(54.8 \%)$ & $4(40.0 \%)$ \\
\hline Yes & $44(47.8 \%)$ & $24(47.1 \%)$ & $20(48.8 \%)$ & $13(44.8 \%)$ & $7(58.3 \%)$ & $14(45.2 \%)$ & $6(60.0 \%)$ \\
\hline Portal vein invasion (n) & & & 0.006 & & 0.156 & & 0.622 \\
\hline No & $86(93.5 \%)$ & $51(100 \%)$ & $35(85.4 \%)$ & $23(79.3 \%)$ & $12(100 \%)$ & $27(87.1 \%)$ & $8(80.0 \%)$ \\
\hline Yes & $6(6.5 \%)$ & 0 & $6(14.6 \%)$ & $6(20.7 \%)$ & 0 & $4(12.9 \%)$ & $2(20.0 \%)$ \\
\hline Bile duct invasion (n) & & & 0.584 & & 1.000 & & 1.000 \\
\hline No & $89(96.7 \%)$ & $50(98.0 \%)$ & $39(95.1 \%)$ & $27(93.1 \%)$ & $12(100 \%)$ & $29(93.5 \%)$ & $10(100 \%)$ \\
\hline Yes & $3(3.3 \%)$ & $1(2.0 \%)$ & $2(4.9 \%)$ & $2(6.9 \%)$ & 0 & $2(6.5 \%)$ & 0 \\
\hline Hepatic vein invasion (n) & & & 1.000 & & 1.000 & & 0.244 \\
\hline No & $89(96.7 \%)$ & $49(96.1 \%)$ & $40(97.6 \%)$ & $28(96.6 \%)$ & $12(100 \%)$ & $31(100 \%)$ & $9(90.0 \%)$ \\
\hline Yes & $3(3.3 \%)$ & $2(3.9 \%)$ & $1(2.4 \%)$ & $1(3.4 \%)$ & 0 & 0 & $1(10.0 \%)$ \\
\hline Microvascular invasion (n) & & & 0.050 & & 0.018 & & 0.292 \\
\hline No & $53(57.6 \%)$ & $34(66.7 \%)$ & $19(46.3 \%)$ & $10(34.5 \%)$ & $9(75.0 \%)$ & $16(51.6 \%)$ & $3(30.0 \%)$ \\
\hline Yes & $39(42.4 \%)$ & $17(33.3 \%)$ & $22(53.7 \%)$ & $19(65.5 \%)$ & $3(25.0 \%)$ & $15(48.4 \%)$ & $7(70.0 \%)$ \\
\hline Tumor stage ${ }^{* * *}$ & & & 0.001 & & 0.315 & & 0.709 \\
\hline pT1 & $73(79.3 \%)$ & $47(92.2 \%)$ & $26(63.4 \%)$ & $16(55.2 \%)$ & $10(83.3 \%)$ & $21(67.7 \%)$ & $5(50.0 \%)$ \\
\hline pT2 & $11(12.0 \%)$ & $1(2.0 \%)$ & $10(24.4 \%)$ & $8(27.6 \%)$ & $2(16.7 \%)$ & $7(22.6 \%)$ & $3(30.0 \%)$ \\
\hline pT3 & $5(5.4 \%)$ & $3(5.9 \%)$ & $2(4.9 \%)$ & $2(6.9 \%)$ & $0(0.0 \%)$ & $1(3.2 \%)$ & $1(10.0 \%)$ \\
\hline pT4 & $3(3.3 \%)$ & $0(0.0 \%)$ & $3(7.3 \%)$ & $3(10.3 \%)$ & $0(0.0 \%)$ & $2(6.5 \%)$ & $1(10.0 \%)$ \\
\hline
\end{tabular}

*Maximum value was set to $40,000 \mathrm{ng} / \mathrm{ml}$. **Maximum value was set to $2,000 \mathrm{mAU} / \mathrm{ml}$. ***Tumor staging according to $7^{\text {th }}$ AJCC tumor staging system. HBV, hepatitis B virus; HCV, hepatitis C virus; ALC, alcoholic liver disease; MELD, model for end-stage liver disease; AFP, alpha-fetoprotein; PIVKA, protein induced by vitamin $\mathrm{K}$ absence or antagonist 
18 months. The median RFP for the early recurrence group was 5.0 months (in a range of 1-16 months), and that of the corresponding late recurrence group was 39.5 months (in a range of 24-86 months). The AFP ( $p=0.037$ ), tumor differentiation $(p=0.003)$, and microvascular invasion ( $p=0.018$ ) were found to be significantly associated with early recurrence according to the univariate analysis (Table 2). However, tumor differentiation $(p=0.043)$ was the sole independent risk factor according to the multivariate analysis (Table 4).

When the RFP for early recurrence was set again to within 10 months, 21 patients (51.2\%) had an early recurrence with the median RFP of 3 months (in a range

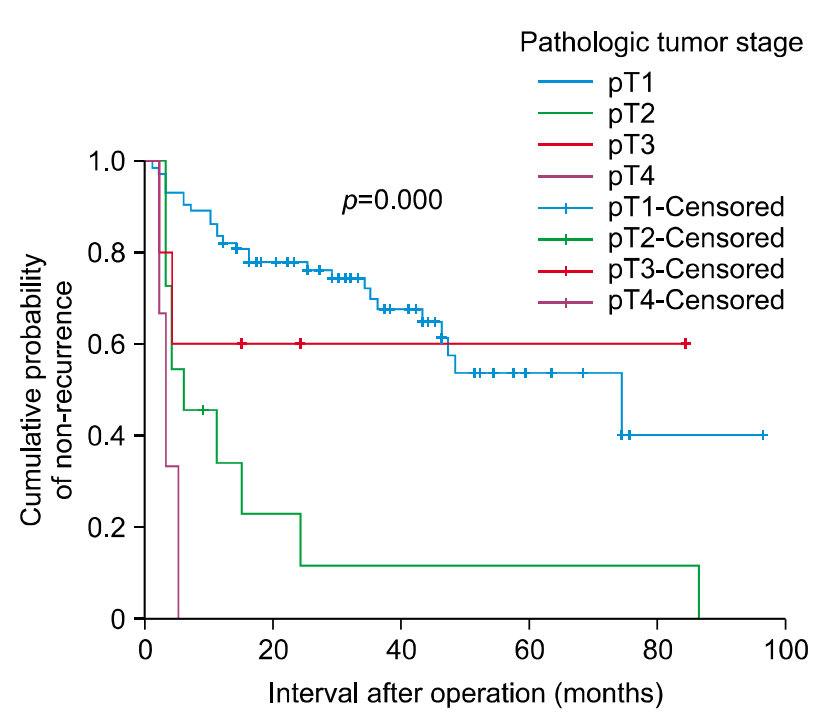

Fig. 1. Comparison of postoperative recurrence-free survival curves, according to the pathological tumor staging.

\section{A}

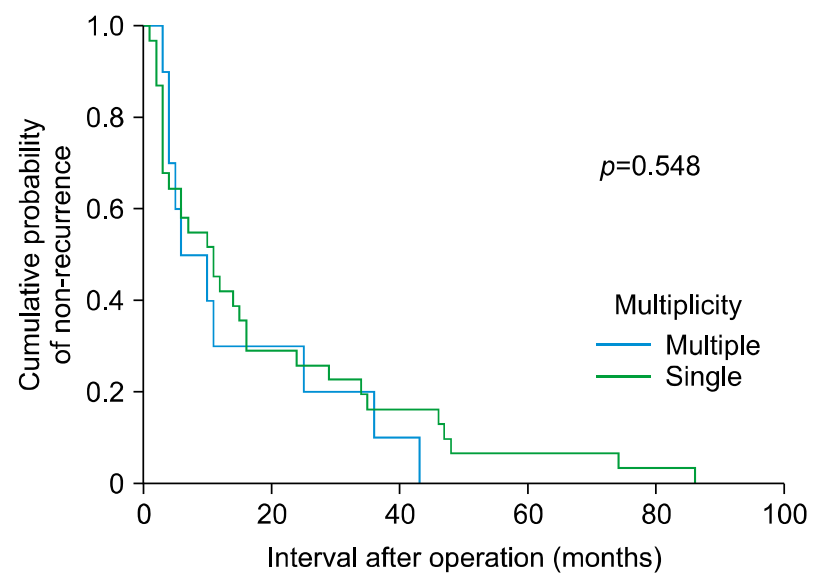

of 1-10 months), and the median RFP for the late recurrence group was 27 months (in a range of 11-86 months). Differentiation $(p=0.004)$, portal vein invasion $(p=0.021)$, and microvascular invasion $(p=0.003)$ were significant risk factors according to the univariate analysis (data not presented). In the multivariate analysis, tumor differentiation $(p=0.038)$ was also the only independent risk factor.

Table 3. Factors of recurrence identified by the multivariate analysis (Cox proportional hazard model)

\begin{tabular}{lrrrr}
\hline \multirow{2}{*}{ Factors } & \multirow{2}{*}{$\begin{array}{c}\text { Hazard } \\
\text { ratio }\end{array}$} & \multicolumn{2}{c}{$95.0 \%$ CI } & \\
\cline { 3 - 4 } & & Lower & Upper & \\
& & & & 0.001 \\
Child-Pugh score & & & & \\
5 vs. 6 & 4.715 & 1.994 & 11.151 & 0.000 \\
5 vs. 7 & 0.134 & 0.012 & 1.554 & 0.108 \\
Portal vein invasion & 7.667 & 2.163 & 27.181 & 0.002 \\
pT stage & & & & 0.001 \\
pT1 vs. pT2 & 6.228 & 2.163 & 18.280 & 0.001 \\
pT1 vs. pT3 & 1.498 & 0.334 & 6.725 & 0.598 \\
pT1 vs. pT4 & 7.476 & 1.775 & 31.484 & 0.006 \\
\hline
\end{tabular}

CI, confidence interval; pT stage, pathologic tumor stage

Table 4. Factors of early recurrence identified by the multivariate analysis ( $\leq 18$ months, logistic regression analysis)

\begin{tabular}{|c|c|c|c|c|}
\hline \multirow{2}{*}{ Factors } & \multirow{2}{*}{$\begin{array}{l}\text { Odds } \\
\text { ratio }\end{array}$} & \multicolumn{2}{|c|}{$95.0 \% \mathrm{CI}$} & \multirow{2}{*}{ - $p$-value } \\
\hline & & Lower & Upper & \\
\hline Worst differentiation & & & & 0.043 \\
\hline Edmondson grade II vs. III & 0.249 & 0.015 & 4.242 & 0.337 \\
\hline Edmondson grade II vs. IV & 0.021 & 0.001 & 0.559 & 0.021 \\
\hline
\end{tabular}

CI, confidence interval

B

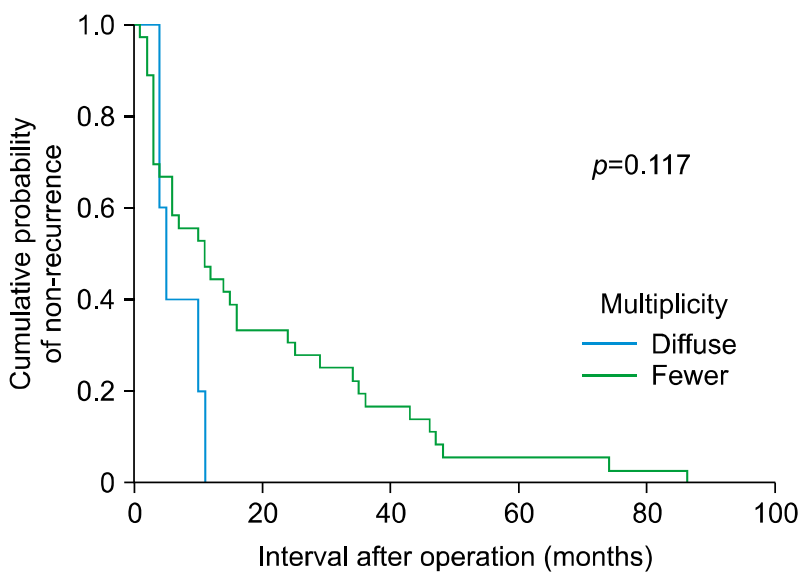

Fig. 2. Comparison of postoperative recurrence-free survival curves, between groups of single versus multiple recurrent nodules $(\mathrm{A})$, and fewer $(\leq 3)$ versus diffuse $(>3)$ recurrent nodules $(\mathrm{B})$. 


\section{Risk factor analysis for multiple tumor recurrences}

Ten patients $(24.4 \%)$ experienced multiple recurrences at first detection. According to the univariate analysis, the AFP $(p=0.002)$, tumor size $(p=0.017)$, tumor necrosis $(p=0.012)$, and hemorrhage $(p=0.022)$ significantly predicted multiple recurrence, but the multivariate analysis failed to identify any independent risk factor.

The median RFP for multiple recurrence was 6.0 months (in a range of 3-43 months), while the median RFP for single recurrence (31 patients, $75.6 \%$ ) was 11.0 months, (in a range of 1-86 months, $p=0.584$, Fig. 2A). When a cutoff of tumor number 3 was applied, there were 36 patients $(87.8 \%)$ in the fewer subgroup (nodule number

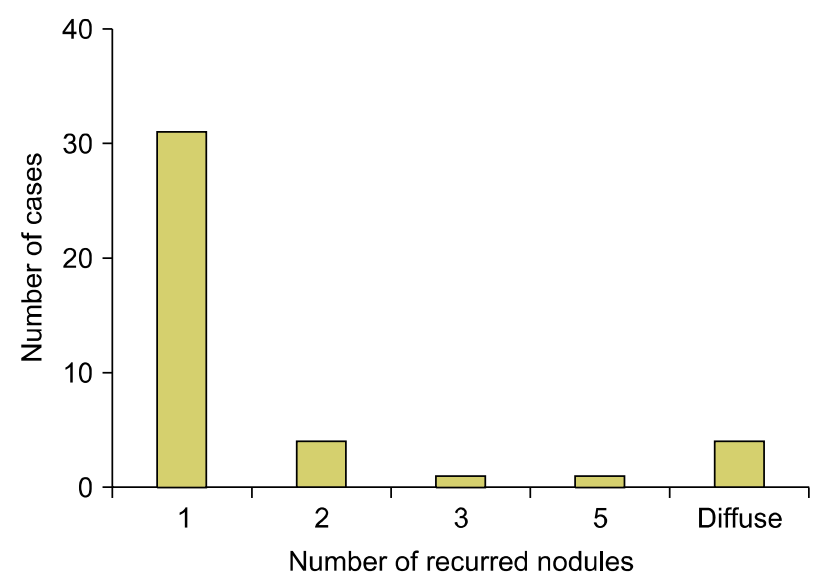

Fig. 3. Distribution of the number of recurrent tumor nodules in the 41 patients with $\mathrm{HCC}$ recurrence. $\leq 3$ ), and five patients (12.2\%) in diffuse (nodule number $>3)$ at first recurrence. A univariate analysis showed the model of end-stage liver disease (MELD) score $(p=0.036)$; the AFP $(p=0.003)$, gross type $(p=0.026)$, and tumor necrosis $(p=0.011)$ were significant risk factors (data not shown), but no independent risk factor was identified by the multivariate analysis. Although the median RFP was significantly shorter in the diffuse subgroup than in the fewer subgroup (5.0 vs. 11.0 months, $p=0.003$ ) according to the Student's t-test, this significance was not confirmed by the log-rank test ( $p=0.117$, Fig. $2 \mathrm{~B}$ ). The distributions of the recurrent nodule numbers are presented in Fig. 3.

\section{Comparison of risk factors for tumor recurrence}

A summary of the risk factors for recurrence, early recurrence, and multiple recurrences are presented in Table 5. No single factor was found to predict the risks of recurrence, early recurrence, and multiple recurrences. Although portal vein invasion was found to be a strong predictor of recurrence, it only predicted early recurrence when a cutoff of 10 months was used. Likewise, although differentiation was found to be an independent risk factor of early recurrence regardless of the RFP criterion, it did not predict recurrence per se, or multiple recurrences. Also, different risk factors were identified by a univariate analysis in each recurrence group, when different criteria were applied. However, no independent risk factor was found to predict recurrence in the multiple recurrence group.

Table 5. Comparison of the risk factors of recurrence in the early/late subgroups (with cutoffs of 18 months or 10 months), and the nodule multiplicity subgroups (with cutoffs of 1 or 3 nodules)

\begin{tabular}{|c|c|c|c|c|c|}
\hline \multirow[b]{2}{*}{ Factors } & \multirow[b]{2}{*}{ Recurrence } & \multicolumn{2}{|c|}{ Early recurrence } & \multicolumn{2}{|c|}{ Multiple recurrences } \\
\hline & & $\leq 18 \mathrm{mos}$ & $\leq 10 \mathrm{mos}$ & $\begin{array}{l}\text { Single vs. } \\
\text { multiple }\end{array}$ & $\begin{array}{c}\text { Fewer }(\leq 3) \text { vs. } \\
\text { Diffuse }(>3)\end{array}$ \\
\hline Child-Pugh score & Yes* & No & No & No & No \\
\hline MELD score & No & No & No & No & Yes \\
\hline AFP & No & Yes & No & Yes & Yes \\
\hline Tumor size & Yes & No & No & Yes & No \\
\hline Gross type & No & No & No & No & Yes \\
\hline Differentiation & No & Yes* & Yes* & No & No \\
\hline Tumor necrosis & Yes & No & No & Yes & Yes \\
\hline Hemorrhage & No & No & No & Yes & No \\
\hline Portal vein invasion & Yes* & No & Yes & No & No \\
\hline Microvascular invasion & No & Yes & Yes & No & No \\
\hline Tumor stage & Yes* & No & No & No & No \\
\hline
\end{tabular}

*Independent risk factors. AFP, alpha-fetoprotein; MELD, model for end-stage liver disease 


\section{DISCUSSION}

Although the prognosis for HCC patients has been improved by the establishment of various management and follow-up strategies, especially those based on considerations of viral hepatitis activity and liver cirrhosis severity, long term outcomes are compromised by intra- and extrahepatic recurrence. ${ }^{5,14}$ In this study, the intrahepatic recurrence rate was $44.6 \%$, which is lower than most of those previously reported. ${ }^{3,5}$ The possible cause of the lower recurrence rate observed in this study is that macroscopic and microscopic satellite lesions, which are significant predictors of intrahepatic recurrence, were excluded. ${ }^{12}$

Intrahepatic recurrence has been reported to outnumber extrahepatic recurrence by six to seven fold and, ${ }^{15}$ thus, intrahepatic recurrence has been the subject of extensive study. ${ }^{5}$ However, the majority of studies on potential risk factors compared the treatment modalities and/or recurrence patterns, and the reported results vary considerably. Furthermore, the incongruity of the risks identified seems to be due to the different criteria applied, the discriminatory powers of the statistical methods used, and the diversity of the causative agents and patient population. ${ }^{16,17}$ This study shows that changing group criteria, even in the same patient population, results in the identification of different risk factors. ${ }^{4}$ More specifically, the risk factors of early recurrence were changed by simply modifying the RFP criterion from within 18 months to within 10 months. Likewise, when the recurrence multiplicity cutoff was altered from $>1$ to $>3$, different risk factors were identified by the univariate analysis. These results suggest that recurrence, timing, and multiplicity are driven by different factors. ${ }^{5}$

The tumor size at the initial presentation has been relatively consistently identified as a risk factor of intrahepatic recurrence, ${ }^{4,18}$ and this study confirms tumor size as a risk factor along with portal vein invasion, which together constitute the tumor $(\mathrm{T})$ stage as defined by the International Union for Cancer Control (UICC). ${ }^{4,20}$ However, when a risk factor analysis for intrahepatic recurrence was performed versus extrahepatic recurrence, rather than non-recurrence, these significances disappeared. ${ }^{3,4,15}$ The Child-Pugh score, as an independent risk factor of intrahepatic recurrence, means that liver cirrhosis is a risk factor. ${ }^{4}$ Cumulative evidence suggests that the primary tumor burden (as indicated by the tumor size and the AFP), as well as the degree of liver cirrhosis (as indicated by the Child-Pugh score and the indocyanine green retention test) are the major conditions that provoke the intrahepatic recurrence of $\mathrm{HCC}$, irrespective of the initial management modality. ${ }^{6,721-23}$ Of note, the width and involvement of the resection margins and methods (anatomical resection versus partial hepatectomy), which are long debated issues in the context of recurrence, were not identified as risk factors in this study. ${ }^{18,24}$

When HCC recurrence was subdivided into early and late recurrence, using an RFP cutoff of 18 months, the risk factors identified differed from those of recurrence per $s e$. In this study, tumor differentiation was an independent risk factor of early recurrence for RFP cutoffs of 18 and 10 months, and tumor differentiation is known to be related to tumor invasiveness; ${ }^{15}$ however, tumor size and portal vein invasion were not found to be risk factors, even by the univariate analysis. On the other hand, Shirabe et al. $^{8}$ reported that capsule invasion, a tumor location deep in the liver parenchyma, and portal vein invasion independently predicted early HCC recurrence. These discrepancies between the identified risk factors may be due to the definitions of early recurrence used, and the different study populations. ${ }^{25}$ Although the term 'early' is non-specific, discrimination is required to define the timing of recurrent $\mathrm{HCC}$, because synchronous metastasis has a poorer prognosis than metachronous occurrence. ${ }^{26}$ Some authors have defined synchronous and metachronous recurrences, using modes of recurrence like intrahepatic metastasis and multicentric occurrence, respectively, and not time courses. ${ }^{27}$ We do not agree with the use of the same definitions for timing and modes of recurrence, and hope that further studies (based on tumor nucleic acid sequencing) contribute to the refining of the terminologies used. $^{28}$

In our series, the serum AFP level, tumor size, tumor necrosis, and hemorrhage were significant risk factors for multiple recurrence, according to the univariate analysis. When a multiplicity cut off of $>3$ was used, rather than a cutoff of $>1$, the MELD score and the gross type replaced the tumor size and hemorrhage. Interestingly, the former factors are related to tumor mass, ${ }^{29}$ while the latter are indicative of liver cirrhosis and tumor invasiveness, respectively. ${ }^{12,30}$ Our failure to identify independent risk 
factors in this study may have been due to the limited number of cases, especially in the diffuse recurrence subgroup, but it might also have been due to the complex relationships between the risk factors. ${ }^{31}$ The distribution of the number of recurrent nodules among the 41 patients had two peaks at one nodule, and for diffuse recurrence throughout the remnant liver. Many authors have set the cutoff number for multiple recurrences to $>3$ nodules, ${ }^{6,10,11,29}$ and we found that counting more than five nodules is impractical. Whatever number criterion is used to define multiplicity, the presence of two peaks in the nodule number distribution indicates that there are distinct groups with different etiologies and prognoses. ${ }^{11,12,29}$

There were some limitations to this study. Since this was a small-volume retrospective study reported by a single center, high-volume multicenter studies need to be performed to validate our results. We presented only the recurrence-free survival results in this study, to avoid complexity of data presentation; thus, the overall patient survival results were omitted.

In conclusion, our results imply that different factors can predict recurrence, timing, and multiplicity of HCC recurrence. Further studies should be conducted to prove the complex relationships between tumor burden, invasiveness, and underlying liver cirrhosis of initial tumors, as well as the timing and multiplicity of recurrent HCC.

\section{ACKNOWLEDGEMENTS}

This work was supported by the Inha University Research Grant.

\section{REFERENCES}

1. Bodzin AS, Busuttil RW. Hepatocellular carcinoma: advances in diagnosis, management, and long term outcome. World J Hepatol 2015;7:1157-1167.

2. Korean Liver Cancer Study Group (KLCSG); National Cancer Center, Korea (NCC). 2014 Korean Liver Cancer Study Group-National Cancer Center Korea practice guideline for the management of hepatocellular carcinoma. Korean J Radiol 2015; 16:465-522.

3. Byeon J, Cho EH, Kim SB, Choi DW. Extrahepatic recurrence of hepatocellular carcinoma after curative hepatic resection. Korean J Hepatobiliary Pancreat Surg 2012;16:93-97.

4. Schlitt HJ, Neipp M, Weimann A, Oldhafer KJ, Schmoll E, Boeker K, et al. Recurrence patterns of hepatocellular and fibrolamellar carcinoma after liver transplantation. J Clin Oncol 1999; 17:324-331.
5. Colecchia A, Schiumerini R, Cucchetti A, Cescon M, Taddia M, Marasco G, et al. Prognostic factors for hepatocellular carcinoma recurrence. World J Gastroenterol 2014;20:5935-5950.

6. Kim GM, Choi GH, Han DH, Kim DH, Kang CM, Choi JS, et al. The risk factors for the intrahepatic recurrence of hepatocellular carcinoma after curative resection. Korean J Hepatobiliary Pancreat Surg 2008;12:222-231.

7. Yoon MH, Choi YI, Park KK, Shin DH, Lee CH. Risk factors for intrahepatic recurrence. Korean J Hepatobiliary Pancreat Surg 2011;15:83-89.

8. Shirabe K, Kanematsu T, Matsumata T, Adachi E, Akazawa K, Sugimachi K. Factors linked to early recurrence of small hepatocellular carcinoma after hepatectomy: univariate and multivariate analyses. Hepatology 1991;14:802-805.

9. Kwon SK, Yun SS, Kim HJ, Lee DS. The risk factors of early recurrence after hepatectomy in hepatocellular carcinoma. Ann Surg Treat Res 2014;86:283-288.

10. Nagasue N, Yukaya H, Chang YC, Yamanoi A, Kohno H, Hayashi T, et al. Assessment of pattern and treatment of intrahepatic recurrence after resection of hepatocellular carcinoma. Surg Gynecol Obstet 1990;171:217-222.

11. Shirabe K, Wakiyama S, Gion T, Motomura K, Koyanagi T, Sakamoto $\mathrm{S}$, et al. Clinicopathological risk factors linked to recurrence pattern after curative hepatic resection for hepatocellular carcinoma--results of 152 resected cases. Hepatogastroenterology 2007;54:2084-2087.

12. Sonoyama T, Ochiai T, Hironaka T, Yamagishi H. Predictors of postoperative diffuse intrahepatic recurrence of hepatocellular carcinoma. Hepatogastroenterology 2003;50:1078-1084.

13. Kawano Y, Sasaki A, Kai S, Endo Y, Iwaki K, Uchida H, et al. Prognosis of patients with intrahepatic recurrence after hepatic resection for hepatocellular carcinoma: a retrospective study. Eur J Surg Oncol 2009;35:174-179.

14. Attwa MH, El-Etreby SA. Guide for diagnosis and treatment of hepatocellular carcinoma. World J Hepatol 2015;7:1632-1651.

15. Taketomi A, Toshima T, Kitagawa D, Motomura T, Takeishi K, Mano Y, et al. Predictors of extrahepatic recurrence after curative hepatectomy for hepatocellular carcinoma. Ann Surg Oncol 2010; $17: 2740-2746$.

16. Naito S, Imamura H, Tukada A, Matsuyama Y, Yoshimoto J, Sugo H, et al. Postoperative recurrence pattern and prognosis of patients with hepatocellular carcinoma, with particular reference to the hepatitis viral infection status. Liver Int 2014;34:802-813.

17. Wakai T, Shirai Y, Yokoyama N, Nagakura S, Hatakeyama K. Hepatitis viral status affects the pattern of intrahepatic recurrence after resection for hepatocellular carcinoma. Eur J Surg Oncol 2003;29:266-271.

18. Marubashi S, Gotoh K, Akita H, Takahashi H, Sugimura K, Miyoshi N, et al. Analysis of recurrence patterns after anatomical or non-anatomical resection for hepatocellular carcinoma. Ann Surg Oncol 2015;22:2243-2252.

19. Subramaniam S, Kelley RK, Venook AP. A review of hepatocellular carcinoma (HCC) staging systems. Chin Clin Oncol 2013;2:33.

20. Park SK, Jung YK, Chung DH, Kim KK, Park YH, Lee JN, et al. Factors influencing hepatocellular carcinoma prognosis after hepatectomy: a single-center experience. Korean J Intern Med 2013;28:428-438.

21. Ng KK, Poon RT, Lo CM, Yuen J, Tso WK, Fan ST. Analysis of recurrence pattern and its influence on survival outcome after radiofrequency ablation of hepatocellular carcinoma. J Gastrointest Surg 2008;12:183-191.

22. Pompili M, Rapaccini GL, de Luca F, Caturelli E, Astone A, Siena DA, et al. Risk factors for intrahepatic recurrence of hep- 
atocellular carcinoma in cirrhotic patients treated by percutaneous ethanol injection. Cancer 1997;79:1501-1508.

23. An SL, Xiao T, Wang LM, Rong WQ, Wu F, Feng L, et al. Prognostic significance of preoperative serum alpha- fetoprotein in hepatocellular carcinoma and correlation with clinicopathological factors: a single-center experience from China. Asian Pac J Cancer Prev 2015;16:4421-4427.

24. Poon RT, Fan ST, Ng IO, Wong J. Significance of resection margin in hepatectomy for hepatocellular carcinoma: a critical reappraisal. Ann Surg 2000;231:544-551.

25. Zhou YM, Yang JM, Li B, Yin ZF, Xu F, Wang B, et al. Risk factors for early recurrence of small hepatocellular carcinoma after curative resection. Hepatobiliary Pancreat Dis Int 2010;9: 33-37.

26. Yamamoto $M$, Matsuda $M$, Iimuro $Y$, Fujii $H$, Nagahori $K$, Ainota T. Intrahepatic distant metastasis and metachronous multicentric occurrence in solitary hepatocellular carcinoma of less than five centimeters in diameter. Surg Today 1993;23:969-978.

27. Matsuda M, Fujii H, Kono H, Matsumoto Y. Surgical treatment of recurrent hepatocellular carcinoma based on the mode of re- currence: repeat hepatic resection or ablation are good choices for patients with recurrent multicentric cancer. J Hepatobiliary Pancreat Surg 2001;8:353-359.

28. Li SL, Su M, Peng T, Xiao KY, Shang LM, Xu BH, et al. Clinicopathologic characteristics and prognoses for multicentric occurrence and intrahepatic metastasis in synchronous multinodular hepatocellular carcinoma patients. Asian Pac J Cancer Prev 2013;14:217-223.

29. Takeishi K, Maeda T, Tsujita E, Yamashita Y, Harada N, Itoh $\mathrm{S}$, et al. Predictors of intrahepatic multiple recurrences after curative hepatectomy for hepatocellular carcinoma. Anticancer Res 2015;35:3061-3066.

30. Tsujita E, Yamashita Y, Takeishi K, Matsuyama A, Maeda T, Tsutsui S, et al. The clinicopathological impact of gross classification on solitary small hepatocellular carcinoma. Hepatogastroenterology 2013;60:1726-1730.

31. Shah SA, Tan JC, McGilvray ID, Cattral MS, Levy GA, Greig $\mathrm{PD}$, et al. Does microvascular invasion affect outcomes after liver transplantation for HCC? A histopathological analysis of 155 consecutive explants. J Gastrointest Surg 2007;11:464-471. 